\title{
Pandemic-induced Reductions on Swim Training Volume and Performance in Collegiate Swimmers
}

\author{
Gloria Martinez Perez ${ }^{1}$, Matthew VanSumeren' ${ }^{1}$, Michael Brown ${ }^{1}$, Tamara Hew-Butler ${ }^{1 *}$
}

$1 \quad{ }^{1}$ Kinesiology, Health, and Sport Studies, Wayne State University, Detroit, Michigan, 48202 USA; gloria.martinez@wayne.edu; msvansum@wayne.edu; mbrown17@wayne.edu; tamara.hew-butler@wayne.edu * Correspondence: tamara.hew-butler@wayne.edu; Tel.: 313-577-8130

\begin{abstract}
The COVID-19 pandemic caused significant training disruptions during the 2020-21 season due to lockdowns, quarantines, and strict adherence to pandemic protocols. The main purpose of this study was to determine how pandemic training restrictions affected training volume and performance in one collegiate swim team. Cumulative training volume data, across a 28-week season, were compared between a pandemic (2020-2021) versus non-pandemic (2019-2020) season. Swimmers were categorized into three groups (Sprinters, Mid-Distance, and Long-Distance) based on training group. Performance times in 25 swimmers who competed in Regional Championships, during both the non-pandemic and pandemic year, were compared via 1-way ANOVA. 26 male and 22 female swimmers commenced the 2020-21 (pandemic) season, with 23\% of swimmers voluntarily opting out. Three COVID-19 cases were confirmed (2\%) by the medical staff with no longterm effects. Significant reductions in average swim volume were verified in Sprinters $(32,867 \pm 10,135 \mathrm{vs} .14,800 \pm 7,995$ yards; $<<0.001)$,Mid-Distance

$(26,457 \pm 10,692$ vs.17,054 $\pm 9.923 y$ ards; $p<0.001), \quad$ and $\quad$ Long-Distance $\quad(37,600 \pm 14,430$ vs.22,254 $\pm 14,418$ yards; $<<0.001$ ) swimmers (non-pandemic vs. pandemic season, respectively). In the Regional performance analyses, the Sprinters swam faster ( $\mathrm{n}=8 ;-0.5 \pm 0.6 \mathrm{secs})$, while Mid-Distance $(\mathrm{n}=10 ; 0.17 \pm 2.1 \mathrm{secs}) \quad$ and Long-Distance $(\mathrm{n}=7 ; 6.0 \pm 4.9 \mathrm{secs})$ swimmers swam slower $\left(\mathrm{F}=11.76 ; \mathrm{p}=0.0003 ; \mathrm{r}^{2}=0.52\right)$. Thus, the pandemic caused significant reductions in swim training volume, with Sprinters performing better and Long-Distance swimmers performing worse at Regional Championships.
\end{abstract}

Keywords: Swimming, COVID-19, swim performance

\section{Introduction}

For collegiate athletes, the coronavirus disease 2019 (COVID-19) pandemic altered training and competition [1,2]. Team outbreaks [3], unplanned lockdowns [2], frequent quarantines [4] and delays associated with routine testing, masking, and social distance protocols $[4,5]$ severely undermined both training quality and quantity. Moreover, athlete-specific health risks associated with competitive exercise included development of myocarditis [6], increased transmission with close contact sports [7,8], and/or augmented infection risk due to excessive exercise-induced immunosuppression [9]. However, these hypothesized risks to athletes remain complex and controversial $[7,10,11]$.

Current data on pandemic-induced restrictions on athlete health, swim training, and performance remains sparse. Cancellation of the U.S. National Collegiate Athletic Association (NCAA) championships, most major Federation Internationale de Natation (FINA) swimming events [2], and postponement of the Tokyo Olympics [12] in 2020, forced elite swimmers to train alone and often at home [2]. Serendipitously, forced reductions in swim training volume on performance indirectly provided insight on the quality versus quantity debate which suggest that elite level swimmers may not require high swim volumes to perform well [13-16]. 
Thus, the primary aims of this retrospective, observational, study was to 1) determine how pandemic-induced training restrictions affected training volume in a NCAA Division 2 (NCAA D2) swim team (compared with a non-pandemic year) and 2) evaluate differences in swim performance on swimmers who competed at the NCAA D2 Regional Championship meet during both a pandemic (2019-2020) versus a non-pandemic (20202021) year. A secondary aim of this study compared the top- 16 swimming performances, for all swimming events, held at both the Regional and National NCAA D2 meets during a pandemic versus a non-pandemic season. We hypothesized that pandemic-induced restrictions in training volume would result in decreased swimming performances at all levels of competition (i.e., at the local, regional, and national competitive level).

\section{Materials and Methods}

\subsection{Data from a single local swim team}

For the primary aims of this study, observational and retrospective data were collected from the official training logs obtained from the swimming coaches from a single NCAA D2 swim team, at a midwestern university located within the United States (U.S.). Cumulative and average training volume data, across a 28 -week season, were compared between a pandemic (2020-2021) versus a non-pandemic (2019-2020) competitive season. We report training volume (i.e., swimming distance) in yards (yd) as the NCAA Championship events are conducted and reported in yards, rather than meters. Swim training volume data were de-identified, prior to analysis, and the study protocol was reviewed by Wayne State University's IRB and found to qualify for an Exemption according to category 4 (IRB-21-10-4075-B3 Expedited/Exempt-EXEMPT, approval date October 14, 2021).

The local swim team data were further divided into three groups (males and females combined): Sprinters (50yd - 100yd), Mid-Distance (200yd - 400yd), and Long-Distance $(500 \mathrm{yd}-1650 \mathrm{yd})$ swimmers, according to their assigned training groups for each season. Swim training volumes were analyzed as a cumulative average (28-weeks, from September through March) between the pandemic (2020-2021) versus non-pandemic (2019-2020) year. Swim training volume was compared, during the pandemic versus non-pandemic years, using 2-way ANOVA (swim training group as the column factor and pandemic versus non-pandemic years as the row factor).

To assess swimming performance, the finishing times (reported in seconds) for 25 local University swimmers who competed in the Great Lakes Intercollegiate Athletics Conference (GLIAC) Regional Championships, during both the pandemic and non-pandemic years, were evaluated. Swim performance data were analyzed as the change $(\Delta)$ value between the pandemic minus non-pandemic swim performance time. We compared performance times, by training group, using 1-way ANOVA.

\subsection{Data obtained from Regional and National Championship meets}

For the secondary aims of this study, we compared the (publicly available) top 16 swimming performance times, for all events, achieved at both the GLIAC Regional $[17,18]$ and NCAA D2 $[19,20]$ Championships during the pandemic (2020-2021) versus non-pandemic years (2019-2020 for the GLIAC and 2018-2019 for the NCAA meet, as NCAA Championships were cancelled in 2020). We used t-tests to evaluate statistically significant differences in performance (as a mean of the top 16 performance times) between the pandemic and closest non-pandemic year.

All data are reported as means $\pm S D$, with statistical significance set a priori at $\mathrm{p}<0.05$.

\section{Results}

The University remained fully online from March 2020 - September 2021. However, select sports teams (including swimming) were allowed to return to campus in September 2020 (Fall semester) to commence training for competition. Pandemic-induced restrictions for the swim team included frequent (3x/week) COVID-19 testing via nasopharynx swabs, tested for the severe acute respiratory syndrome coronavirus 2 (SARS-CoV-2) virus via polymerase chain reaction (PCR), for all swimmers and coaches participating in the 
competitive season. Swimmers with a positive test or exposed to COVID-19 were required to quarantine for 10 days. Only 8 swimmers were allowed in the pool at one time (unlimited swimmers were allowed in the pool during non-pandemic times), due to social distancing protocols.

Overall, 26 male and 22 female swimmers started the 2020-21 (pandemic) season, with six males (23\%) and five females (23\%) voluntarily opting out by the end of the season. During a typical non-pandemic year (like 2019-20) no swimmers voluntarily dropout. Three total COVID-19 cases were confirmed (2\%) by the medical staff, during the 28week pandemic season, with no long-term effects on athlete health or performance following COVID-19 recovery. The three confirmed COVID-19 cases were associated with mild (1) or no (2) symptoms. For the 37 swimmers who completed the 28 -week pandemic season, a total of ten competitions were either rescheduled, or cancelled. In a typical nonpandemic year, swimmers typically compete in 8-9 number of meets, prior to the regional championship competition.

\subsection{Swim Training and performance results from a single local swim team}

There were significant reductions in average weekly swim volume in Sprinters, MidDistance, and Long-Distance swimmers (non-pandemic vs. pandemic season, respectively) during the 28-week training period (Figure 1).
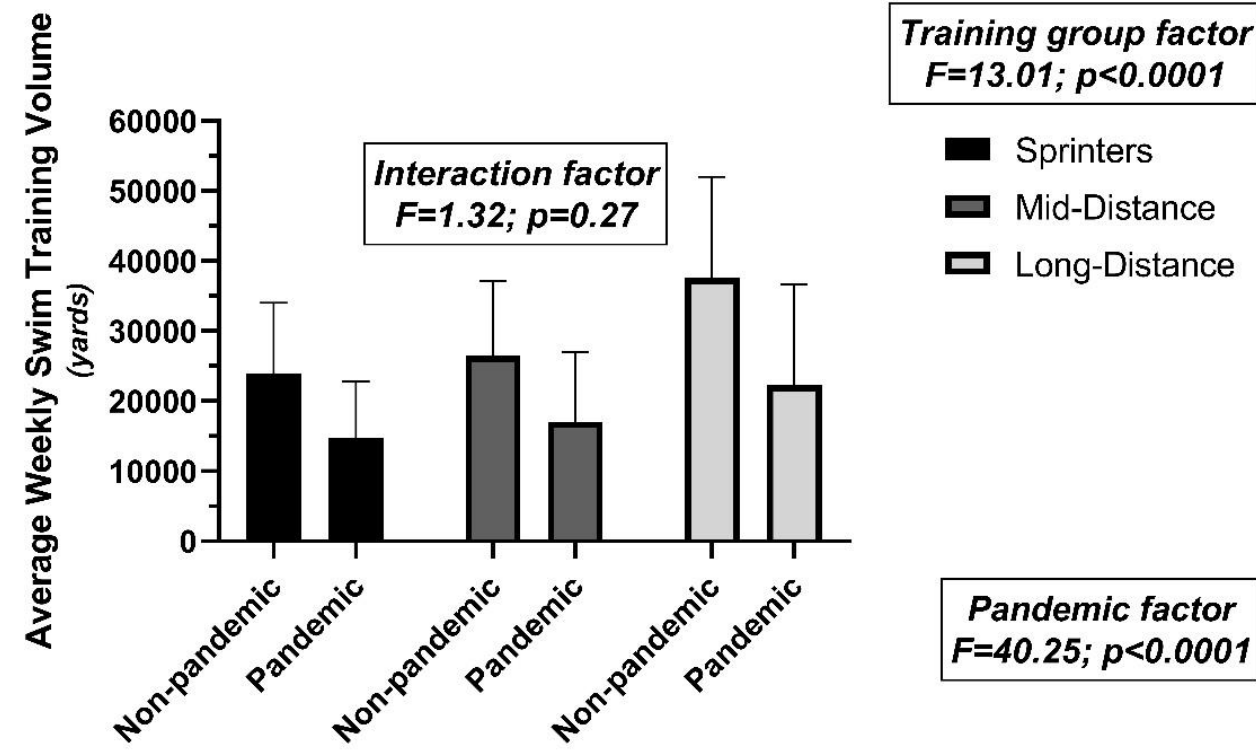

Figure 1: Comparisons of average weekly training swim volumes for Sprinters, Mid-Distance, and Long-Distance swimmers (Training group) during both the non-pandemic and pandemic (Pandemic factor) years.

Cumulative training distance was reduced (non-pandemic vs. pandemic year) in Sprinters (668,275 vs. 414,400 yd; 62\%), Mid-Distance (740,800 vs. 477,500 yd; 64\%), and Long-Distance (1,052,800 vs. 623,100 yd; 59\%) swimmers.

For the 25 swimmers who competed in Regional Championships, in both the pandemic and non-pandemic years, the Sprinters swam faster ( $n=8 ;-0.5 \pm 0.6$ secs), while MidDistance $(n=10 ; 0.17 \pm 2.1$ secs $)$ and Long-Distance $(n=7 ; 6.0 \pm 4.9$ secs $)$ swimmers swam slower $\left(\mathrm{F}=11.76 ; \mathrm{p}=0.0003 ; \mathrm{r}^{2}=0.52\right)$ during the pandemic year compared with the previous non-pandemic year. Of note, improved performance times of the Sprinters occurred despite significant reductions in training volume (Figure 2). 


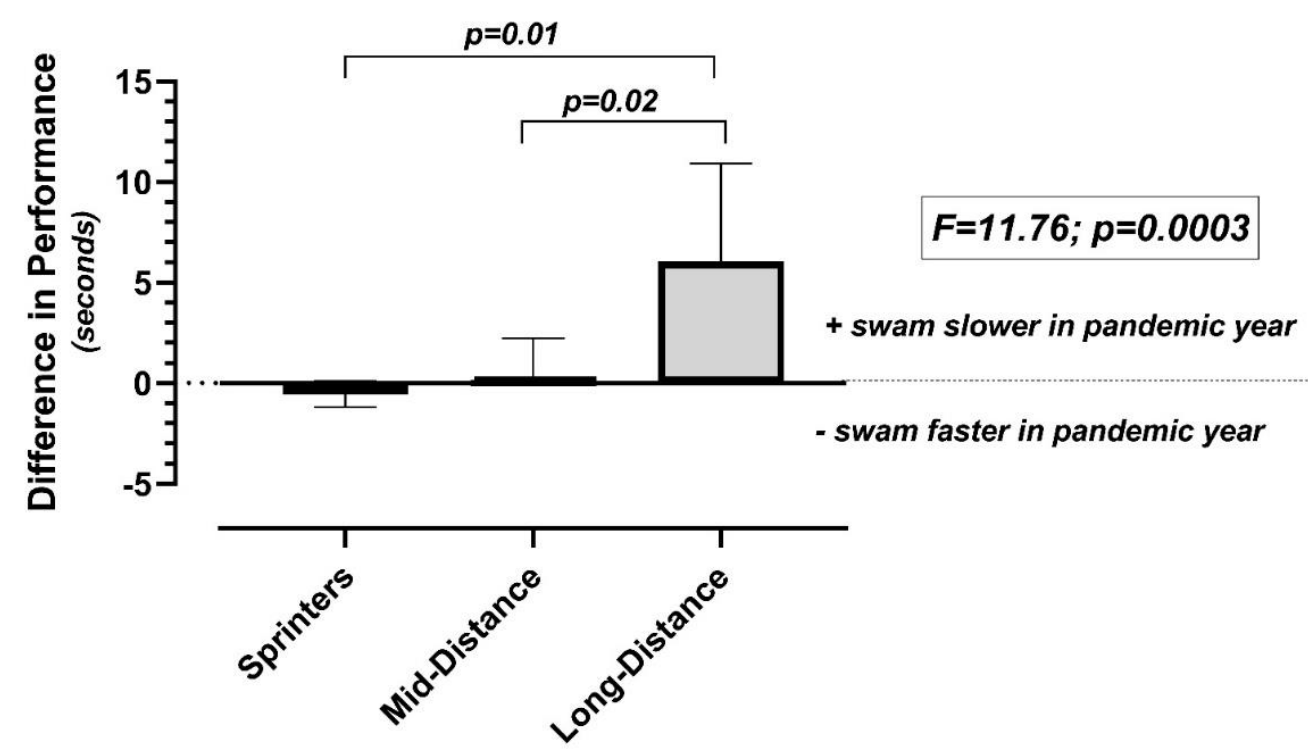

Figure 2: Comparison of performance in the GLIAC regional championship meet, in swimmers competing in both the pandemic and non-pandemic years, categorized by training group.

3.2. Performance Results from the Regional and National Championship meets

In the GLIAC regional swim championship meet, the female swimmers swam significantly slower (as a mean value) in the 200yd freestyle (FR), 500yd FR, 200yd butterfly (FL), 100yd backstroke (BK), and 200yd individual medley (IM) in the pandemic (2020-21) versus non-pandemic (2019-2020) year (Table 1).

Table 1. Top 16 female swimming performances in the GLIAC Regional Championship meets, in a non-pandemic versus pandemic year. All swim events are reported in yards $(y d)$ while all finishing times are reported in seconds. 


\begin{tabular}{|c|c|c|}
\hline Swim Event & $\begin{array}{l}\text { Performance times } \\
\text { Non-pandemic } \\
(2019-2020) \\
\text { Mean } \pm \text { SD } \\
\text { (fastest - slowest) }\end{array}$ & $\begin{array}{l}\text { Performance times } \\
\text { Pandemic } \\
(2020-2021) \\
\text { Mean } \pm \text { SD } \\
\text { (fastest }- \text { slowest) }\end{array}$ \\
\hline $50 \mathrm{FR}$ & $\begin{array}{c}24.3 \pm 0.3 \\
(23.8-24.8)\end{array}$ & $\begin{array}{c}24.3 \pm 0.4 \\
(23.6-24.9)\end{array}$ \\
\hline $100 \mathrm{FR}$ & $\begin{array}{c}52.9 \pm 0.9 \\
(51.4-53.8) \\
\end{array}$ & $\begin{array}{c}52.8 \pm 0.5 \\
(51.9-53.5) \\
\end{array}$ \\
\hline $200 \mathrm{FR}$ & $\begin{array}{c}{ }^{*} 114.4 \pm 1.2 \\
(111.3-115.7)\end{array}$ & $\begin{array}{c}115.5 \pm 1.6 \\
(112.9-117.5)\end{array}$ \\
\hline $500 \mathrm{FR}$ & $\begin{array}{c}* 307.3 \pm 5.1 \\
(298.5-315.9)\end{array}$ & $\begin{array}{c}311.2 \pm 5.7 \\
(298.5-318.1)\end{array}$ \\
\hline $1000 \mathrm{FR}$ & $\begin{array}{c}627.1 \pm 13.2 \\
(605.6-649.2)\end{array}$ & $\begin{array}{c}636.3 \pm 14.7 \\
(603.8-654.3)\end{array}$ \\
\hline $1650 \mathrm{FR}$ & $\begin{array}{c}1111.3 \pm 228.1 \\
(1011.6-1961.4)\end{array}$ & $\begin{array}{c}1080.9 \pm 40.9 \\
(1013.4-1165.7)\end{array}$ \\
\hline $100 \mathrm{FL}$ & $\begin{array}{c}57.7 \pm 1.23 \\
(55.5-59.1) \\
\end{array}$ & $\begin{array}{c}58.4 \pm 1.4 \\
(56.8-60.5)\end{array}$ \\
\hline $200 \mathrm{FL}$ & $\begin{array}{c}{ }^{*} 130 \pm 3.4 \\
(123.4-134.5) \\
\end{array}$ & $\begin{array}{c}135.1 \pm 7.7 \\
(123.3-154.2) \\
\end{array}$ \\
\hline $100 \mathrm{BK}$ & $\begin{array}{c}* 57.7 \pm 1.0 \\
(55.5-58.8)\end{array}$ & $\begin{array}{c}58.7 \pm 1.4 \\
(55.7-61.0) \\
\end{array}$ \\
\hline $200 \mathrm{BK}$ & $\begin{array}{c}126.5 \pm 4.1 \\
(119.7-132.7)\end{array}$ & $\begin{array}{c}129.3 \pm 4.3 \\
(119.9-136.1)\end{array}$ \\
\hline $100 \mathrm{BR}$ & $\begin{array}{c}66.0 \pm 1.3 \\
(63.6-68.0)\end{array}$ & $\begin{array}{c}66.4 \pm 1.6 \\
(63.1-70.3) \\
\end{array}$ \\
\hline $200 \mathrm{BR}$ & $\begin{array}{c}145.4 \pm 4.2 \\
(139.3-151.3) \\
\end{array}$ & $\begin{array}{c}146.4 \pm 3.9 \\
(139.5-152.1) \\
\end{array}$ \\
\hline $200 \mathrm{IM}$ & $\begin{array}{c}{ }^{* *} 128.0 \pm 2.1 \\
(123.7-130.4)\end{array}$ & $\begin{array}{c}130.1 \pm 2.4 \\
(125.7-133.6)\end{array}$ \\
\hline $400 \mathrm{IM}$ & $\begin{array}{c}276.9 \pm 6.6 \\
(268.4-290.5) \\
\end{array}$ & $\begin{array}{c}284.8 \pm 16.5 \\
(269.2-314.4) \\
\end{array}$ \\
\hline
\end{tabular}

${ }^{*} p<0.05 ;{ }^{* *} p<0.01 ;{ }^{* *} p<0.001$ between non-pandemic vs. pandemic average swim performance times $F R=$ Freestyle, $F L=$ Butterfly, $B K=$ Backstroke, $B R=$ Breaststroke, $I M=$ Individual Medley

In the GLIAC Regional Swim Championship meet, the male swimmers swam significantly slower (as a mean value) in the 500yd FR in the pandemic (2020-21) versus nonpandemic (2019-2020) year (Table 2).

Table 2. Top 16 male swimming performances in the GLIAC Regional Championship meets, in a non-pandemic versus pandemic year. All swim events are reported in yards (yd) while all finishing times are reported in seconds 


\begin{tabular}{|c|c|c|}
\hline Swim Event & $\begin{array}{l}\text { Performance times } \\
\text { Non-pandemic } \\
(2019-2020) \\
\text { Mean } \pm \text { SD } \\
\text { (fastest }- \text { slowest) }\end{array}$ & $\begin{array}{l}\text { Performance times } \\
\text { Pandemic } \\
(2020-2021) \\
\text { Mean } \pm \text { SD } \\
\text { (fastest }- \text { slowest) }\end{array}$ \\
\hline $50 \mathrm{FR}$ & $\begin{array}{c}20.8 \pm 0.3 \\
(20.2-21.3)\end{array}$ & $\begin{array}{c}20.8 \pm 0.5 \\
(19.7-21.3)\end{array}$ \\
\hline $100 \mathrm{FR}$ & $\begin{array}{c}45.7 \pm 0.6 \\
(44.7-46.5)\end{array}$ & $\begin{array}{c}46.0 \pm 0.9 \\
(43.9-46.9)\end{array}$ \\
\hline $200 \mathrm{FR}$ & $\begin{array}{c}101.1 \pm 1.3 \\
(98.7-103.3)\end{array}$ & $\begin{array}{c}102.1 \pm 1.5 \\
(98.9-104.0) \\
\end{array}$ \\
\hline $500 \mathrm{FR}$ & $\begin{array}{c}* 276.1 \pm 3.8 \\
(271.4-281.5)\end{array}$ & $\begin{array}{c}280.2 \pm 5.4 \\
(272.1-289.1) \\
\end{array}$ \\
\hline $1000 \mathrm{FR}$ & $\begin{array}{c}597.0 \pm 97.2 \\
(550.4-959.8)\end{array}$ & $\begin{array}{c}577.2 \pm 16.1 \\
(540.5-594.6)\end{array}$ \\
\hline $1650 \mathrm{FR}$ & $\begin{array}{c}964.4 \pm 21.2 \\
(923.0-992.3) \\
\end{array}$ & $\begin{array}{c}979.3 \pm 33.4 \\
(916.5-1034.1) \\
\end{array}$ \\
\hline $100 \mathrm{FL}$ & $\begin{array}{c}50.0 \pm 0.7 \\
(47.8-50.7)\end{array}$ & $\begin{array}{c}50.2 \pm 0.9 \\
(48.0-51.3)\end{array}$ \\
\hline $200 \mathrm{FL}$ & $\begin{array}{c}114.1 \pm 2.1 \\
(109.8-116.6)\end{array}$ & $\begin{array}{c}114.0 \pm 3.2 \\
(108.7-117.8)\end{array}$ \\
\hline $100 \mathrm{BK}$ & $\begin{array}{c}51.0 \pm 1.4 \\
(48.2-53.6)\end{array}$ & $\begin{array}{c}50.9 \pm 1.3 \\
(48.1-52.8)\end{array}$ \\
\hline $200 \mathrm{BK}$ & $\begin{array}{c}114.7 \pm 6.2 \\
(106.8-128.2)\end{array}$ & $\begin{array}{c}112.7 \pm 3.6 \\
(105.9-117.5)\end{array}$ \\
\hline $100 \mathrm{BR}$ & $\begin{array}{c}57.7 \pm 1.8 \\
(54.9-61.0)\end{array}$ & $\begin{array}{c}57.1 \pm 1.8 \\
(54.1-59.9)\end{array}$ \\
\hline $200 \mathrm{BR}$ & $\begin{array}{c}125.6 \pm 2.9 \\
(120.2-130.0)\end{array}$ & $\begin{array}{c}126.7 \pm 4.0 \\
(120.4-132.7)\end{array}$ \\
\hline $200 \mathrm{IM}$ & $\begin{array}{c}113.1 \pm 2.2 \\
(108.9-115.5)\end{array}$ & $\begin{array}{c}113.5 \pm 2.2 \\
(109.2-115.9)\end{array}$ \\
\hline $400 \mathrm{IM}$ & $\begin{array}{c}246.0 \pm 5.5 \\
(237.2-253.9)\end{array}$ & $\begin{array}{c}247.6 \pm 7.4 \\
(235.2-258.0)\end{array}$ \\
\hline
\end{tabular}

${ }^{*} p<0.05 ;{ }^{* *} p<0.01 ;{ }^{* * *} p<0.001$ between pandemic vs. non-pandemic average swim performance times $F R=$ Freestyle, $F L=$ Butterfly, $B K=$ Backstroke, $B R=$ Breaststroke, $I M=$ Individual Medley

In the NCAA D2 National Swim Championship meet, the female swimmers swam significantly slower (as a mean value) in the 50yd FR and 100yd FR while swimming significantly faster in the 400yd IM in the pandemic (2020-21) versus non-pandemic (201819) year (Table 3).

Table 3. Top 16 female swimming performances in the NCAA D2 National Championship meets, in a non-pandemic versus pandemic year. All swim events are reported in yards (yd) while all finishing times are reported in seconds. 


\begin{tabular}{|c|c|c|}
\hline Swim Event & $\begin{array}{l}\text { Performance times } \\
\text { Non-pandemic } \\
(2018-2019) \\
\text { Mean } \pm \text { SD } \\
\text { (fastest - slowest) }\end{array}$ & $\begin{array}{c}\text { Performance times } \\
\text { Pandemic } \\
(2020-2021) \\
\text { Mean } \pm \text { SD } \\
\text { (fastest }- \text { slowest) }\end{array}$ \\
\hline $50 \mathrm{FR}$ & $\begin{array}{l}{ }^{* *} 23.1 \pm 0.3 \\
(22.4-23.4) \\
\end{array}$ & $\begin{array}{c}23.4 \pm 0.2 \\
(22.8-23.6)\end{array}$ \\
\hline $100 \mathrm{FR}$ & $\begin{array}{l}* * 50.2 \pm 0.7 \\
(48.4-50.8) \\
\end{array}$ & $\begin{array}{c}50.8 \pm 0.5 \\
(50.0-51.4)\end{array}$ \\
\hline $200 \mathrm{FR}$ & $\begin{array}{c}109.5 \pm 0.8 \\
(108.0-110.4)\end{array}$ & $\begin{array}{c}110.0 \pm 0.8 \\
(108.4-110.9)\end{array}$ \\
\hline $500 \mathrm{FR}$ & $\begin{array}{c}294.6 \pm 1.8 \\
(291.6-297.0)\end{array}$ & $\begin{array}{c}295.7 \pm 2.3 \\
(291.8-299.2)\end{array}$ \\
\hline $1000 \mathrm{FR}$ & $\begin{array}{c}603.4 \pm 8.1 \\
(585.9-613.7) \\
\end{array}$ & $\begin{array}{c}608.2 \pm 7.3 \\
(593.2-618.6)\end{array}$ \\
\hline $1650 \mathrm{FR}$ & $\begin{array}{c}1010.9 \pm 14.4 \\
(980.1-1023.5)\end{array}$ & $\begin{array}{c}1012.9 \pm 14.1 \\
(991.0-1034.1)\end{array}$ \\
\hline $100 \mathrm{FL}$ & $\begin{array}{c}54.5 \pm 0.8 \\
(52.5-55.4)\end{array}$ & $\begin{array}{c}54.8 \pm 0.8 \\
(53.0-56.0)\end{array}$ \\
\hline $200 \mathrm{FL}$ & $\begin{array}{c}121.5 \pm 0.9 \\
(120.0-122.8)\end{array}$ & $\begin{array}{c}122.6 \pm 2.1 \\
(119.1-126.1)\end{array}$ \\
\hline $100 \mathrm{BK}$ & $\begin{array}{c}54.6 \pm 0.8 \\
(52.5-55.5) \\
\end{array}$ & $\begin{array}{c}55.1 \pm 0.9 \\
(52.9-56.2) \\
\end{array}$ \\
\hline $200 \mathrm{BK}$ & $\begin{array}{c}119.2 \pm 1.5 \\
(116.1-120.9)\end{array}$ & $\begin{array}{c}119.7 \pm 1.3 \\
(116.6-121.6)\end{array}$ \\
\hline $100 \mathrm{BR}$ & $\begin{array}{c}62.2 \pm 0.7 \\
(60.5-62.9)\end{array}$ & $\begin{array}{c}62.6 \pm 0.4 \\
(62.0-63.4)\end{array}$ \\
\hline $200 \mathrm{BR}$ & $\begin{array}{c}135.9 \pm 1.7 \\
(132.1-138.0)\end{array}$ & $\begin{array}{c}136.6 \pm 1.8 \\
(133.0-139.1)\end{array}$ \\
\hline $200 \mathrm{IM}$ & $\begin{array}{c}121.7 \pm 1.4 \\
(118.8-123.8)\end{array}$ & $\begin{array}{c}122.5 \pm 1.8 \\
(119.1-124.7)\end{array}$ \\
\hline $400 \mathrm{IM}$ & $\begin{array}{c}* 262.6 \pm 2.8 \\
(255.1-265.6)\end{array}$ & $\begin{array}{c}259.8 \pm 3.7 \\
(254.2-264.7)\end{array}$ \\
\hline
\end{tabular}

${ }^{*} p<0.05 ;{ }^{* *} p<0.01 ;{ }^{* *} p<0.001$ between pandemic vs. non-pandemic average swim performance times $F R=$ Freestyle,$F L=$ Butterfly, $B K=$ Backstroke, $B R=$ Breaststroke, $I M=$ Individual Medley

In the NCAA D2 National Swim Championship meet, the male swimmers swam significantly slower (as a mean value) in the 200yd BK while swimming significantly faster in the 50yd FR in the pandemic (2020-21) versus non-pandemic (2018-19) year (Table 4).

Table 4. Top 16 male swimming performances in the NCAA D2 National Championship meets, in a non-pandemic versus pandemic year. All swim events are reported in yards $(y d)$ while all finishing times are reported in seconds. 


\begin{tabular}{|c|c|c|}
\hline Swim Event & $\begin{array}{l}\text { Performance times } \\
\text { Non-pandemic } \\
(2018-2019) \\
\text { Mean } \pm \text { SD } \\
\text { (fastest - slowest) }\end{array}$ & $\begin{array}{l}\text { Performance times } \\
\text { Pandemic } \\
(2020-2021) \\
\text { Mean } \pm \text { SD } \\
\text { (fastest }- \text { slowest) }\end{array}$ \\
\hline $50 \mathrm{FR}$ & $\begin{array}{l}{ }^{* * 20.0 \pm 0.2} \\
(19.6-20.3)\end{array}$ & $\begin{array}{c}19.8 \pm 0.2 \\
(19.3-20.0)\end{array}$ \\
\hline $100 \mathrm{FR}$ & $\begin{array}{c}43.8 \pm 0.3 \\
(43.1-44.2) \\
\end{array}$ & $\begin{array}{c}43.7 \pm 0.4 \\
(43.0-44.2) \\
\end{array}$ \\
\hline $200 \mathrm{FR}$ & $\begin{array}{c}96.8 \pm 0.9 \\
(94.0-97.5)\end{array}$ & $\begin{array}{c}96.7 \pm 1.5 \\
(93.3-98.3)\end{array}$ \\
\hline $500 \mathrm{FR}$ & $\begin{array}{c}266.3 \pm 1.2 \\
(264.5-268.1)\end{array}$ & $\begin{array}{c}266.4 \pm 2.1 \\
(263.1-269.4)\end{array}$ \\
\hline $1000 \mathrm{FR}$ & $\begin{array}{c}552.1 \pm 6.7 \\
(536.8-560.6) \\
\end{array}$ & $\begin{array}{c}553.6 \pm 6.9 \\
(534.1-561.0) \\
\end{array}$ \\
\hline $1650 \mathrm{FR}$ & $\begin{array}{c}927.0 \pm 10.1 \\
(906.4-939.9)\end{array}$ & $\begin{array}{c}928.4 \pm 12.1 \\
(895.1-941.6)\end{array}$ \\
\hline $100 \mathrm{FL}$ & $\begin{array}{c}47.4 \pm 0.7 \\
(45.0-48.1)\end{array}$ & $\begin{array}{c}47.0 \pm 0.7 \\
(45.6-47.9)\end{array}$ \\
\hline $200 \mathrm{FL}$ & $\begin{array}{c}107.0 \pm 1.5 \\
(102.5-108.6)\end{array}$ & $\begin{array}{c}106.9 \pm 1.6 \\
(102.9-108.9)\end{array}$ \\
\hline $100 \mathrm{BK}$ & $\begin{array}{c}47.5 \pm 0.4 \\
(46.7-48.3)\end{array}$ & $\begin{array}{c}47.7 \pm 0.4 \\
(47.0-48.3)\end{array}$ \\
\hline $200 \mathrm{BK}$ & $\begin{array}{c}{ }^{* 105.2} \pm 0.8 \\
(104.0-106.6)\end{array}$ & $\begin{array}{c}105.9 \pm 0.7 \\
(104.5-107.0)\end{array}$ \\
\hline $100 \mathrm{BR}$ & $\begin{array}{c}54.2 \pm 0.6 \\
(52.9-54.8)\end{array}$ & $\begin{array}{c}54.2 \pm 0.7 \\
(52.4-55.0)\end{array}$ \\
\hline $200 \mathrm{BR}$ & $\begin{array}{c}118.8 \pm 1.1 \\
(116.9-120.1)\end{array}$ & $\begin{array}{c}119.0 \pm 1.3 \\
(116.7-121.0)\end{array}$ \\
\hline $200 \mathrm{IM}$ & $\begin{array}{c}107.6 \pm 0.9 \\
(105.6-109.0)\end{array}$ & $\begin{array}{c}107.5 \pm 1.1 \\
(105.1-108.8)\end{array}$ \\
\hline $400 \mathrm{IM}$ & $\begin{array}{c}232.8 \pm 1.7 \\
(230.4-235.6)\end{array}$ & $\begin{array}{c}231.4 \pm 2.7 \\
(225.8-235.2)\end{array}$ \\
\hline
\end{tabular}

${ }^{*} p<0.05 ;{ }^{* *} p<0.01 ;{ }^{* *} p<0.001$ between pandemic vs. non-pandemic average swim performance times $F R=$ Freestyle,$F L=$ Butterfly, $B K=$ Backstroke, $B R=$ Breaststroke, $I M=$ Individual Medley

\section{Discussion}

From the overarching perspective of sports safety, the incidence of COVID-19 was low $(2 \%)$ in our cohort of NCAA D2 swimmers during the 2020-2021 season. Two of the three COVID-19 positive swimmers were asymptomatic (67\%). This finding agrees with a previous study, conducted on a large (10,265 students) collegiate population, which demonstrated that $>50 \%$ of positive COVID-19 cases were without symptoms [21]. Fortunately, all three of our COVID-19 swimmers returned to training and competition without any lingering side-effects. This finding agrees with a previous study conducted on 46 elite Hungarian swimmers, whereas all 14 (30\%) COVID-19 positive swimmers recovered and resumed training for the Tokyo Olympics [12]. Additionally, only $1.2 \%$ of 3529 COVID-19 positive NCAA student-athletes reported lingering symptomatology $>3$ weeks following SARS-CoV-2 infection [22]. Our rigorous COVID-19 testing protocol (COVID19 testing 3x/week) appeared to be effective in reducing the spread of COVID-19 amongst athletes, as similarly verified in a cohort of Southeastern Conference collegiate football players [7]. Most COVID-19 spread amongst athletes seems to occur at social gatherings and communal living arrangements, rather than during official training and competition, $[4,7]$ as anecdotally supported in our swimming cohort.

From a mental health perspective, $\sim 1$ of 4 (23\%) of our swimmers opted (dropped) out of the season during the pandemic year. This drop-out rate was most likely due to enhanced psychological stress, rather than training distress from the pandemic [23,24]. 
A recent (pre-pandemic) systematic review conducted on swimmers supports this assumption, citing common reasons for dropping out due to "pressure" and "lack of fun" [25]. Of note, the most common reasons for dropping out of swimming appear to be within an athlete's control [25]. This suggests that in future pandemics, strategies to maximize an athlete's sense of "control" (through freely available mental health counseling) should be prioritized to minimize student-athlete drop-out rates. Curiously, our drop-out rate was equal between sexes, which conflicts with previous (pre-pandemic) findings which suggest that mental health symptoms are typically higher in female versus male elite athletes $[25,26]$.

\subsection{Swim Training and performance results from a single local swim team}

Pandemic-induced restrictions resulted in significant reductions in overall swim training volumes, in our collegiate swim team, as expected from COVID-19 social distancing protocols, lockdowns, and quarantines [2]. Despite the cumulative decrease in swim training volume during the pandemic, the Sprinters performed better (i.e., swam faster) while the Mid-Distance swimmers performed similarly. The Long-Distance swimmers, however, performed worse (i.e., swam slower). This performance decline in the LongDistance swimmers was expected, as a previous report conducted on 18 professional cyclists documented a $33.9 \%$ decrease in total training volume which resulted in a $1 \%$ and $19 \%$ decrease in 5-minute and 20-minute time-trial performance time, respectively [1]. A typical Long-Distance swim race, similarly, lasts between $\sim 4$ to $\sim 15$ minutes (Tables 1-4).

Our finding that Sprinters performed better with reduced swim training volumes also indirectly informs a larger debate regarding the efficacy of quality versus quantity training on swimmer performance [13-16] and health [27]. A growing body of evidence suggests that high swimming volume does not offer performance advantages over low swim training volume, past adolescence [28]. Furthermore, since $81 \%$ of 32 Olympic pool events cover at or less than $200 \mathrm{~m}$ in total distance, with an average race duration less than 140 seconds, the efficacy of high swimming volumes (aerobic training) on anaerobic sprinting performance appears maladaptive [13-15] in lieu of shorter race-paced training [16]. With additional regards to health risks, high swim training volumes have also been associated with shoulder pain in adolescent swimmers [27] as well as overtraining and burnout [29]. Thus, our results support the training concept that low swim volume training may offer performance advantages to Sprinters but is likely deleterious to Long-Distance swimming performance.

\subsection{Performance Results from the Regional and National Championship meets}

The largest pandemic-induced decreases in average (top-16) performance times were noted in female swimmers competing at the GLIAC Regional Championships, whereas $36 \%$ of races ( 5 of 14 ) recorded significantly slower average racing times during the pandemic compared to the non-pandemic year. For male swimmers competing in the GLIAC Championship meet, only one race $(7 \%)$, the $500 \mathrm{yd}$ FR, recorded a slower average finishing time during the pandemic (versus non-pandemic) year. The reasons why females swam slower during the pandemic year remain unclear and multifactorial. We can only speculate that females, at lower levels of competition, were collectively more susceptible to mental health challenges [25,26], burnout [29], and/or overtraining [30], during the pandemic, which adversely affected their Regional swim performances.

At the NCAA D2 National Championship meet, however, the top finishing times (on average) were not as adversely affected. In some events, like the $400 \mathrm{~m}$ IM for females and 50 FR for males, the swimmers unexpectedly performed faster (as an average) during the pandemic year. This finding, that pandemic-induced restrictions were not deleterious to swim performances at the National championship level is supported by previous data suggesting that short periods of COVID-19-induced detraining did not adversely affect performance in Hungarian swimmers training for the Tokyo Olympics [12]. Thus, the performance effects from the COVID-19 lockdown are higher at the local level than at the elite national level for reasons for reasons yet to be clarified. 


\subsection{Practical Implications}

The practical points obtained from a critical analysis of retrospective, observational, data suggest the following: 1) almost one-quarter ( 23\%) of collegiate NCAA D2 swimmers may likely drop out from official team activities without added interventions (like mental health support); 2) thrice weekly COVID-19 testing appears to limit the spread of COVID-19 amongst competitive swimmers; 3) COVID-19-positive student-athletes do not appear to suffer long-term health or performance consequences; 4) swim training volumes are significantly reduced during a pandemic, which negatively impacts Long-Distance swimming performance; 5) Sprinters appear to perform better with reduced swim training volumes, which supports the quality over quantity debate; and 6) swim performance is least impacted at the highest level of collegiate competition (NCAA championships). Thus, as Michigan currently faces another winter COVID-19 surge, these data serve to inform strategies which reduce the deleterious consequences of a pandemic on studentathlete health, training, and swim performance.

\subsection{Strengths and Limitations}

The strength of these data are that swimming volumes and performance times are routinely recorded and easily reproduceable. Potential confounding effects of the external environment - such as ambient temperature, wind speed, and variable terrain - are relatively controlled at indoor swimming venues regardless of pool location. As such, comparing swim performance times across both time (year) and space (location) represent reliable comparisons (unlike outdoor sporting events). The limitations of these data include our reliance on existing records, as a primary source of data, and inability to quantify the amount of dryland training the swimmers received. Previous studies suggest that dryland training may enhance swimming performance in both a pandemic [31] and nonpandemic [32] times, which may have confounded our swim performance results.

\section{Conclusions}

Reduced, pandemic-induced, swim training volumes positively impacted Sprinting performance while negatively impacting Long-Distance swim performance in a cohort of midwestern NCAA D2 swimmers. Swim performance declines, during the pandemic, were mostly evident in female swimmers competing at Regional championship events. The average swim performance was largely unaffected at the National level (in a pandemic versus non-pandemic year). While a substantial $(23 \%)$ number of swimmers dropped out during the pandemic season, our overall COVID-19 transmission rates were low with all (2\%) COVID-19 positive swimmers recovering without ill-effects.

\footnotetext{
Author contributions: All authors were involved with the development of this article and contributed to the following: conceptualization, GMP, THB; Methodology, GMP, THB; Formal Analysis, THB; Investigation, GMP, MVS, MB, THB; Data Curation, GMP; Writing - Original draft presentation, THB, GMP, MVS, MB; Funding acquisition, none; Project administration, GMP, THB; Writing - Review and Editing, GMP, MVS, MB, THB. All authors read and approved the final version of the manuscript and agreed with the order of presentation of the authors.
}

Funding: This research received no external funding 
Acknowledgements: The authors wish to thank Hunter Steigman for providing information about injuries, COVID-19 health, and recovery and Leonardo de Oliveira for assisting with data acquisition.

Institutional Review Board Statement: The study protocol was reviewed by Wayne State University's IRB and found to qualify for Exemption according to category 4 (IRB-21-10-4075-B3 Expedited/Exempt-EXEMPT, approval date October 14, 2021).

Informed Consent Statement: Informed Consent was not necessary, for this retrospective, observational, study which qualified for Exemption according to category 4.

Data Availability Statement: All data will be available on request to the corresponding author (THB).

Conflict of interest: The authors declare no conflict of interest

\section{References}

1. Muriel, X.; Courel-Ibáñez, J.; Cerezuela-Espejo, V.; Pallarés, J.G. Training Load and Performance Impairments in Professional Cyclists During COVID-19 Lockdown. Int J Sports Physiol Perform 2020, 16, 735-738, doi:10.1123/ijspp.2020-0501.

2. Haddad, M.; Abbes, Z.; Mujika, I.; Chamari, K. Impact of COVID-19 on Swimming Training: Practical Recommendations during Home Confinement/Isolation. Int J Environ Res Public Health 2021, 18, doi:10.3390/ijerph18094767.

3. Teran, R.G., I; Gretsch, S; Cable, T; Black, SR; Green, SJ; Perez, O; Chlipala, GE; Meienschein-Cline, M; Kuntsman, KJ; Bleasdale, SC; Fricchione, MJ. COVID-19 Outbreak Among a University's Men's and Women's Soccer Teams - Chicago, Illinois, July-August 2020. MMWR Morb Mortal Wkly Rep 20020, 69, 1591-1594, doi:http://dx.doi.org/10.15585/mmwr.mm6943e5.

4. $\quad$ Atherstone, C.; Peterson, M.L.; Malone, M.; Honein, M.A.; MacNeil, A.; O'Neal, C.S.; Paul, S.; Harmon, K.G.; Goerl, K.; Wolfe, C.R., et al. Time from Start of Quarantine to SARS-CoV-2 Positive Test Among Quarantined College and University Athletes - 17 States, June-October 2020. MMWR Morb Mortal Wkly Rep 2021, 70, 7-11, doi:10.15585/mmwr.mm7001a2.

5. Lendacki, F.R.; Teran, R.A.; Gretsch, S.; Fricchione, M.J.; Kerins, J.L. COVID-19 Outbreak Among Attendees of an Exercise Facility - Chicago, Illinois, August-September 2020. MMWR Morb Mortal Wkly Rep 2021, 70, 321-325, doi:10.15585/mmwr.mm7009e2.

6. Rajpal, S.; Tong, M.S.; Borchers, J.; Zareba, K.M.; Obarski, T.P.; Simonetti, O.P.; Daniels, C.J. Cardiovascular Magnetic Resonance Findings in Competitive Athletes Recovering From COVID-19 Infection. JAMA Cardiol 2021, 6, 116-118, doi:10.1001/jamacardio.2020.4916.

7. Dixon, B.C.; Fischer, R.S.B.; Zhao, H.; O'Neal, C.S.; Clugston, J.R.; Gibbs, S.G. Contact and SARS-CoV-2 Infections Among College Football Athletes in the Southeastern Conference During the COVID-19 Pandemic. JAMA Netw Open 2021, 4, e2135566, doi:10.1001/jamanetworkopen.2021.35566.

8. Atrubin, D.; Wiese, M.; Bohinc, B. An Outbreak of COVID-19 Associated with a Recreational Hockey Game - Florida, June 2020. MMWR Morb Mortal Wkly Rep 2020, 69, 1492-1493, doi:10.15585/mmwr.mm6941a4.

9. Hew-Butler, T.; Smith-Hale, V.; Van Sumeren, M.; Sabourin, J.; Levy, P. Is Exercise the Best Medicine during a COVID-19 Pandemic? Comment on Constandt, B.; Thibaut, E.; De Bosscher, V.; Scheerder, J.; Ricour, M.; Willem, A. Exercising in Times of Lockdown: An Analysis of the Impact of COVID-19 on Levels and Patterns of Exercise among Adults in Belgium. Int. J. Environ. Res. Public Health 2020, 17, 4144. Int J Environ Res Public Health 2020, 17, doi:10.3390/ijerph17165730.

10. Starekova, J.; Bluemke, D.A.; Bradham, W.S.; Eckhardt, L.L.; Grist, T.M.; Kusmirek, J.E.; Purtell, C.S.; Schiebler, M.L.; Reeder, S.B. Evaluation for Myocarditis in Competitive Student Athletes Recovering From Coronavirus Disease 2019 With Cardiac Magnetic Resonance Imaging. JAMA Cardiol 2021, 6, 945-950, doi:10.1001/jamacardio.2020.7444.

11. Martinez, M.W.; Tucker, A.M.; Bloom, O.J.; Green, G.; DiFiori, J.P.; Solomon, G.; Phelan, D.; Kim, J.H.; Meeuwisse, W.; Sills, A.K., et al. Prevalence of Inflammatory Heart Disease Among Professional Athletes With Prior COVID-19 Infection Who Received Systematic Return-to-Play Cardiac Screening. JAMA Cardiol 2021, 6, 745-752, doi:10.1001/jamacardio.2021.0565. 
12. Csulak, E.; Petrov, Á.; Kováts, T.; Tokodi, M.; Lakatos, B.; Kovács, A.; Staub, L.; Suhai, F.I.; Szabó, E.L.; Dohy, Z., et al. The Impact of COVID-19 on the Preparation for the Tokyo Olympics: A Comprehensive Performance Assessment of Top Swimmers. Int J Environ Res Public Health 2021, 18, doi:10.3390/ijerph18189770.

13. Nugent, F.J.; Comyns, T.M.; Burrows, E.; Warrington, G.D. Effects of Low-Volume, High-Intensity Training on Performance in Competitive Swimmers: A Systematic Review. J Strength Cond Res 2017, 31, 837-847, doi:10.1519/jsc.0000000000001583.

14. Nugent, F.J.; Comyns, T.M.; Warrington, G.D. Quality versus Quantity Debate in Swimming: Perceptions and Training Practices of Expert Swimming Coaches. J Hum Kinet 2017, 57, 147-158, doi:10.1515/hukin-2017-0056.

15. Nugent, F.; Comyns, T.; Nevill, A.; Warrington, G.D. The Effects of Low-Volume, High-Intensity Training on Performance Parameters in Competitive Youth Swimmers. Int J Sports Physiol Perform 2019, 14, 203-208, doi:10.1123/ijspp.2018-0110.

16. Williamson, D.; McCarthy, E.; Ditroilo, M. Acute Physiological Responses to Ultra Short Race-Pace Training in Competitive Swimmers. J Hum Kinet 2020, 75, 95-102, doi:10.2478/hukin-2020-0040.

2020 GLIAC Championships 2/19/2020 to

https:/d2 2figo6ddd0g cloudfront.net/t/1/aumm8bu6rp8bx/gliac complete

2021 GLIAC Championships 2/17/2021 to 2/20/2021. Availabe online:

https://d2o2figo6ddd0g.cloudfront.net/h/1/oohdtd3mgurlb4/mm8results2col.pdf (accessed on November 17, 2021)

19. 2019 NCAA Division II Men's \& Women's Swimming and Diving Championships. 2019, https://www.swimmingworldmagazine.com/results/pdf/2019-ncaa-division-ii-championships-full-results-ncaa-college2019-03-13.pdf, doi:https://www.swimmingworldmagazine.com/results/pdf/2019-ncaa-division-ii-championships-fullresults-ncaa-college-2019-03-13.pdf (accessed on November 17, 2021).

20. NCAA Division II Championships 3/17/2021 - 3/20/2021. Availabe online: https://swimmeetresults.tech/NCAA-Division-II2021/ (accessed on November 17, 2021).

21. Denny, T.N.; Andrews, L.; Bonsignori, M.; Cavanaugh, K.; Datto, M.B.; Deckard, A.; DeMarco, C.T.; DeNaeyer, N.; Epling, C.A.; Gurley, T., et al. Implementation of a Pooled Surveillance Testing Program for Asymptomatic SARS-CoV-2 Infections on a College Campus - Duke University, Durham, North Carolina, August 2-October 11, 2020. MMWR Morb Mortal Wkly Rep 2020, 69, 1743-1747, doi:10.15585/mmwr.mm6946e1.

22. Petek, B.J.; Moulson, N.; Baggish, A.L.; Kliethermes, S.A.; Patel, M.R.; Churchill, T.W.; Harmon, K.G.; Drezner, J.A. Prevalence and clinical implications of persistent or exertional cardiopulmonary symptoms following SARS-CoV-2 infection in 3597 collegiate athletes: a study from the Outcomes Registry for Cardiac Conditions in Athletes (ORCCA). Br J Sports Med 2021, 10.1136/bjsports-2021-104644, doi:10.1136/bjsports-2021-104644.

23. Swami, V.; Horne, G.; Furnham, A. COVID-19-related stress and anxiety are associated with negative body image in adults from the United Kingdom. Pers Individ Dif 2021, 170, 110426, doi:10.1016/j.paid.2020.110426.

24. Ettman, C.K.; Abdalla, S.M.; Cohen, G.H.; Sampson, L.; Vivier, P.M.; Galea, S. Prevalence of Depression Symptoms in US Adults Before and During the COVID-19 Pandemic. JAMA Netw Open 2020, 3, e2019686, doi:10.1001/jamanetworkopen.2020.19686.

25. Monteiro, D.; Cid, L.; Marinho, D.A.; Moutão, J.; Vitorino, A.; Bento, T. Determinants and Reasons for Dropout in Swimming -Systematic Review. Sports (Basel) 2017, 5, doi:10.3390/sports5030050.

26. Walton, C.C.; Rice, S.; Gao, C.X.; Butterworth, M.; Clements, M.; Purcell, R. Gender differences in mental health symptoms and risk factors in Australian elite athletes. BMJ Open Sport Exerc Med 2021, 7, e000984, doi:10.1136/bmjsem-2020-000984. Feijen, S.; Tate, A.; Kuppens, K.; Claes, A.; Struyf, F. Swim-Training Volume and Shoulder Pain Across the Life Span of the Competitive Swimmer: A Systematic Review. J Athl Train 2020, 55, 32-41, doi:10.4085/1062-6050-439-18.

Aspenes, S.T.; Karlsen, T. Exercise-training intervention studies in competitive swimming. Sports Med 2012, 42, 527-543, doi:10.2165/11630760-000000000-00000. 
29. Martin, J.; Byrd, B.; Hew-Butler, T.; Moore, E.W.G. A longitudinal study on the psychological and physiological predictors of burnout in NCAA collegiate swimmers. Journal of Applied Sport Psychology 2021, 10.1080/10413200.2021.1974603, 1-17, doi:10.1080/10413200.2021.1974603.

30. Stanfa, M.R.; Silles, N.N.; Cooper, A.; Arena, S.; Landis-Piwowar, K.; Aprik, C.; Hew-Butler, T. Risk Factors for Collegiate Swimmers Hospitalized With Exertional Rhabdomyolysis. Clin J Sport Med 2017, 27, 37-45.

31. Subekti, R.M.B.H., Y; Novian, G. The Effect of Dry Land Training Model on Youth Athletes Swimming Performance Ducring Covid-19 Pandemic. Journal of Physical Education, Health and Sport 2021, 8, 12-16.

32. Crowley, E.; Harrison, A.J.; Lyons, M. The Impact of Resistance Training on Swimming Performance: A Systematic Review. Sports Med 2017, 47, 2285-2307, doi:10.1007/s40279-017-0730-2. 